\title{
Photon-pressure-induced test mass deformation in gravitational-wave detectors
}

\author{
S Hild ${ }^{1}$, M Brinkmann ${ }^{1}$, K Danzmann ${ }^{1}$, H Grote ${ }^{1}$, M Hewitson ${ }^{1}$, \\ J Hough ${ }^{1}$, H Lück ${ }^{1}$, I Martin ${ }^{2}$, K Mossavi ${ }^{1}$, N Rainer ${ }^{1}$, S Reid ${ }^{2}$, \\ J R Smith ${ }^{3}$, K Strain ${ }^{2}$, M Weinert ${ }^{1}$, P Willems ${ }^{4}$, B Willke ${ }^{1}$ \\ and $\mathrm{W}$ Winkler ${ }^{1}$ \\ ${ }^{1}$ Max-Planck-Institut für Gravitationsphysik (Albert-Einstein-Institut) and Leibniz Universität \\ Hannover, Callinstr 38, D-30167 Hannover, Germany \\ 2 SUPA, Physics \& Astronomy, University of Glasgow, Glasgow G12 8QQ, UK \\ ${ }^{3}$ Syracuse University, Department of Physics, 201 Physics Building, Syracuse, New York \\ 13244-1130, USA \\ ${ }^{4}$ The LIGO project, California Institute for Technology, Mail Stop 18-34, Pasadena, \\ California 91125, USA \\ E-mail: stefan.hild@aei.mpg.de
}

Received 9 August 2007, in final form 28 September 2007

Published 6 November 2007

Online at stacks.iop.org/CQG/24/5681

\begin{abstract}
A widely used assumption within the gravitational-wave community has so far been that a test mass acts like a rigid body for frequencies in the detection band, i.e. for frequencies far below the first internal resonance. In this paper, we demonstrate that localized forces, applied for example by a photon pressure actuator, can result in a non-negligible elastic deformation of the test masses. For a photon pressure actuator setup used in the gravitational-wave detector GEO 600, we measured that this effect modifies the standard response function by $10 \%$ at $1 \mathrm{kHz}$ and about $100 \%$ at $2.5 \mathrm{kHz}$.
\end{abstract}

PACS numbers: $04.80 . \mathrm{Nn}, 95.75 . \mathrm{Kk}$

(Some figures in this article are in colour only in the electronic version)

\section{Introduction}

The currently operating laser-interferometric gravitational-wave detectors GEO 600 [1], Virgo [2], LIGO [3] and TAMA300 [4] are using cylindrical test masses made of fused silica to probe changes in the metric originating from gravitational waves. Usually, such test masses are considered to behave as rigid bodies for any applied force.

However, in this paper, we show that a non-uniformly distributed force acting on a test mass can cause a significant deformation. We evaluate this effect quantitatively, using the 


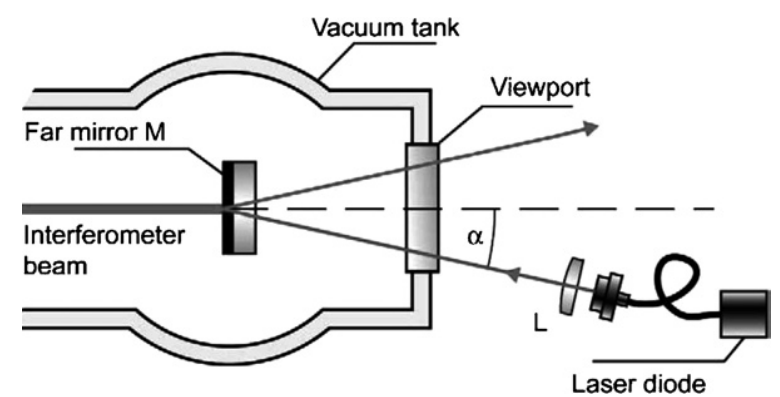

Figure 1. Experimental arrangement for measurements with the photon pressure actuator. M, far mirror in the north building of the GEO 600 detector. Illumination of this mirror with light from the laser diode produces a differential arm-length change that is measured at the main output of the interferometer.

Table 1. Parameters used for calculating the photon-pressure-induced test mass deformation.

\begin{tabular}{ll}
\hline Test mass diameter & $180 \mathrm{~mm}$ \\
Test mass thickness & $100 \mathrm{~mm}$ \\
Test mass material & Fused silica (Suprasil) \\
Beam of photon pressure actuator & $5 \mathrm{~mm}$ diameter (Gaussian) \\
Interferometer beam & $50 \mathrm{~mm}$ diameter (Gaussian) \\
\hline
\end{tabular}

photon pressure actuator installed at the GEO 600 interferometer. Such a photon pressure actuator was first demonstrated by Clubley et al [5] to be employed as an easy and independent method for displacement calibration of a gravitational-wave detector.

Figure 1 shows a diagram of the experimental setup of the photon pressure actuator in the GEO 600 detector. The light of a laser diode is collimated by a lens, L, and enters the vacuum system via a viewport, before it is impinging on one of the main mirrors, M. More detailed descriptions of the setup can be found in $[6,7]$.

In section 2, we calculate the expected test mass deformation induced by the photon pressure using three different methods. Two-dimensional and three-dimensional finite element analyses are performed as well as an analytical estimation. The corresponding displacement sensed by the gravitational-wave detector is evaluated in section 3 using a simple model. A prediction is made of how the response to the photon actuator changes when the test mass deformation effect is taken into account. The presence of the test mass deformation is experimentally confirmed by measurements presented in section 4 .

\section{Models used to calculate the test mass deformation}

In order to evaluate the effect from any test mass deformation introduced by photon pressure, we have to calculate the actual deformation caused by the setup of the GEO photon pressure actuator. Table 1 indicates the parameters used for the calculations.

Altogether we compared three different ways to calculate the mirror deformation: two finite element analyses have been performed in addition to a (quasi-)analytical calculation. The following list gives a short description of each of the methods applied:

- ANSYS: ANSYS is a well-known finite element software package [8]. The threedimensional model consisted of about 42000 elements. A non-homogeneous meshing 


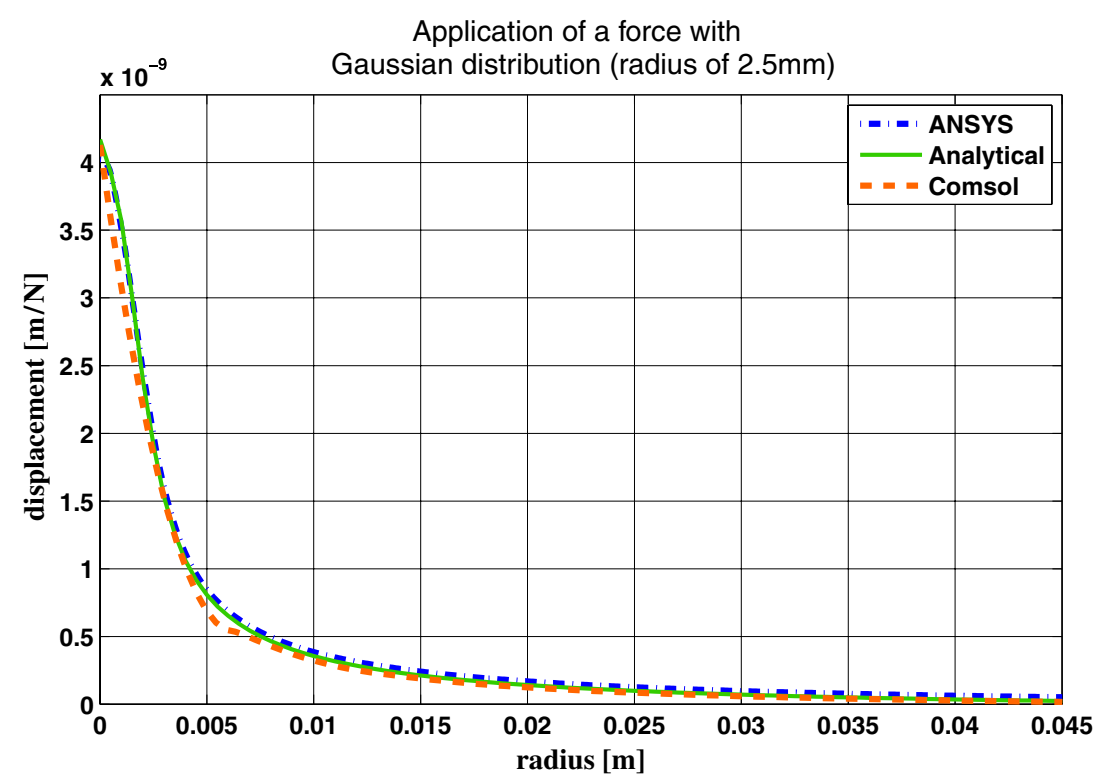

Figure 2. Radial profile of the test mass deformation originating from a force continuously applied with a Gaussian distribution over a radius of $2.5 \mathrm{~mm}$ around the center of the test mass. For the analysis a GEO 600 standard test mass was used $(18 \mathrm{~cm}$ diameter, $10 \mathrm{~cm}$ thickness, made of fused silica (Suprasil)). Shown are the results of two finite element analysis. The result produced by the ANSYS software is represented by the blue dashed-dotted line, while the COMSOL software produced the orange dashed line. An analytical calculation is indicated by the solid green line. The corresponding effective displacements (see table 2) agree within $10 \%$ for all three results. (Explanation is given in the text.)

was chosen, employing a very dense meshing in the center of the test mass in order to resolve the maximum of the deformation.

- Comsol: Comsol multiphysics [9] is also a widely used finite element analysis software package. The deformation of the cross-section of the test mass was analyzed by performing a two-dimensional simulation.

- Analytical: The analytical calculation is based on an algorithm developed by Bondu, Hello and Vinet [10] for the calculation of thermo-elastic noise in an infinite half-plane mirror. In addition, our calculation includes corrections and extensions from Liu and Thorne [11].

Figure 2 shows the radial profile of the calculated displacement of the test mass surface, $D(r)$. The three methods agree pretty well over the full radius of the test mass.

The effective displacement actually measured by the interferometer, $D_{\text {total }}$, is determined by the overlap of the mirror deformation and the main interferometer beam, which can be described by a Gaussian beam. The radial intensity of the interferometer beam, $I(r)$, is given by

$$
I(r)=\exp \left(\frac{-2 r^{2}}{\omega^{2}}\right),
$$

where $\omega=25 \mathrm{~mm}$ is the radius of the beam. Each point on the mirror surfaces contributes to the total effective displacement, $D_{\text {total }}$, weighted by the product of the power of the 
Table 2. Results of the three different calculations of the photon-pressure-induced test mass deformation. The three values for the effective displacement agree to within $10 \%$ and the resulting notch frequencies (see section 3) match within $5 \%$.

\begin{tabular}{lll}
\hline & $\begin{array}{l}\text { Effective } \\
\text { displacement }\left(\mathrm{m} \mathrm{N}^{-1}\right)\end{array}$ & $\begin{array}{l}\text { Resulting notch } \\
\text { frequency }(\mathrm{Hz})\end{array}$ \\
\hline ANSYS & $3.83 \times 10^{-10}$ & 3598 \\
Comsol & $3.49 \times 10^{-10}$ & 3768 \\
Analytical & $3.54 \times 10^{-10}$ & 3741 \\
\hline
\end{tabular}

main interferometer beam, $I(r)$, and the corresponding displacement $D(r) .^{5}$ For a radially symmetric setup, as described above, the total effective displacement can be expressed by a single integral

$D_{\text {total }}=\int_{0}^{0.09 \mathrm{~m}} \int_{0}^{2 \pi} D_{\text {eff }} \cdot r \cdot \mathrm{d} r \cdot \mathrm{d} \varphi=\int_{0}^{0.09 \mathrm{~m}} 2 \pi \cdot r \cdot k_{I} \cdot I(r) \cdot D(r) \cdot \mathrm{d} r$.

The factor $k_{I}$ is a normalization factor for $I(r)$ in order to give

$$
\int_{0}^{0.09 \mathrm{~m}} 2 \pi \cdot r \cdot k_{I} \cdot I(r) \cdot \mathrm{d} r=1 .
$$

Using equation (2) the total effective displacement for all three calculated test mass deformations can be derived. The corresponding results are displayed in the second column of table 2. For the three different calculations of the test mass deformation the effective displacement sensed by the main interferometer, $D_{\text {total }}$, agrees within about $10 \%$.

\section{Predicted effect of the photon-pressure-induced test mass deformation}

The previous section showed that the test masses are not completely rigid and that the photon pressure actuator beam really causes a non-negligible deformation. Next, we have to evaluate how strong the displacement originating from the non-rigidity of the test mass is, compared to the displacement of the center of mass (originating from the pendulum response). Since both responses are linear in the applied light power of the photon pressure actuator we can simply compare their responses.

The pendulum response, $\alpha_{\text {pen }}$, follows a $1 / f^{2}$-law, has a magnitude of $5 \times 10^{-7} \mathrm{~m} \mathrm{~N}^{-1}$ at $100 \mathrm{~Hz}$ and is $180^{\circ}$ out of phase with the light power modulation (see blue dashed trace figure 3$)^{6}$. The response of the mirror deformation, $\alpha_{\text {def }}$, is assumed to be flat in frequency and in phase with the modulated light power for frequencies below the first internal resonances of the test mass, which in the case of a GEO test mass, has a frequency of roughly $11 \mathrm{kHz}$ [12]. In the previous section, effective displacements in the range $3.54-3.83 \times 10^{-10} \mathrm{~m} \mathrm{~N}^{-1}$ were found. The magnitude and phase of $\alpha_{\text {def }}$ from the analytical test mass deformation is shown by the red dashed-dotted trace in figure 3 . The total response, $\alpha_{\text {total }}$, plotted in green (solid) is the sum of the two individual responses:

$$
\alpha_{\text {total }}=\alpha_{\text {pen }}+\alpha_{\text {def }} .
$$

5 The phase change of the interferometer light due to the mirror deformation is proportional to the light amplitude $\sqrt{I(r)}$. At the sensing point of the interferometer (which is a photodiode) the signal is beaten with the local oscillator, which also has a spatial profile of $\sqrt{I(r)}$. Taking both these effects into account we have to use $I(r)$ for weighting the radial test mass deformation $D(r)$.

6 Due to the fact that we are only interested in frequencies far above the resonance of the pendulum which is around $1 \mathrm{~Hz}$, excitation and pendulum motion have opposite phase. 

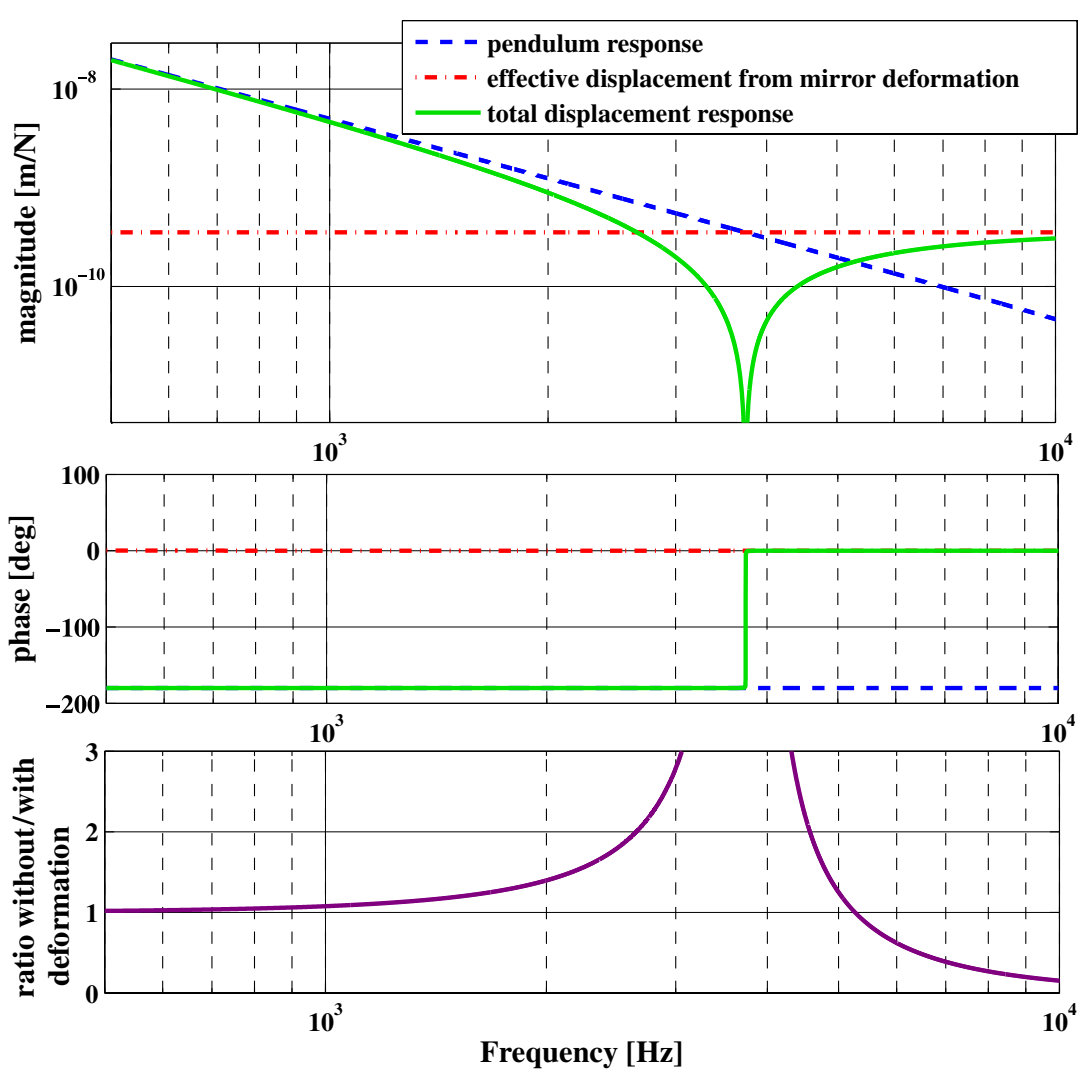

Figure 3. Simple model for the photon pressure calibrator taking into account the responses from the pendulum and from the mirror deformation effect (analytical). The pendulum response follows a $1 / f^{2}$-law and is $180^{\circ}$ out of phase from the photon pressure excitation. The mirror deformation has a flat response and is in phase with the photon pressure actuation. If both responses are added a notch appears at the frequency where both responses have equal size. The purple trace shows the expected discrepancy between the calibrations with and without accounting for the test mass deformation.

The total response shows a steep notch at the frequency where the individual responses have the same magnitude and compensate each other completely due to having opposite phase. At the frequency of the notch the phase of $\alpha_{\text {total }}$ jumps from -180 to $0^{\circ}$. The modeled notch frequencies for the three different methods are displayed in the third column of table 2. The resulting discrepancy between the photon pressure actuator response without and with test mass deformation is shown in the lowest subplot of figure 3 . Already at $1 \mathrm{kHz}$ the test mass deformation causes an effect of $10 \%$. At $2 \mathrm{kHz}$ the error, when not taking the test mass deformation into account, amounts to $40 \%$ and between $2.6 \mathrm{kHz}$ and $4.6 \mathrm{kHz}$ the discrepancy is larger than $100 \%$.

\section{Measurement of the photon-pressure-induced test mass deformation}

In order to verify this model, we injected signals to the photon pressure actuator over a wide frequency range including frequencies as high as $6 \mathrm{kHz}$ to be able to resolve any potential 

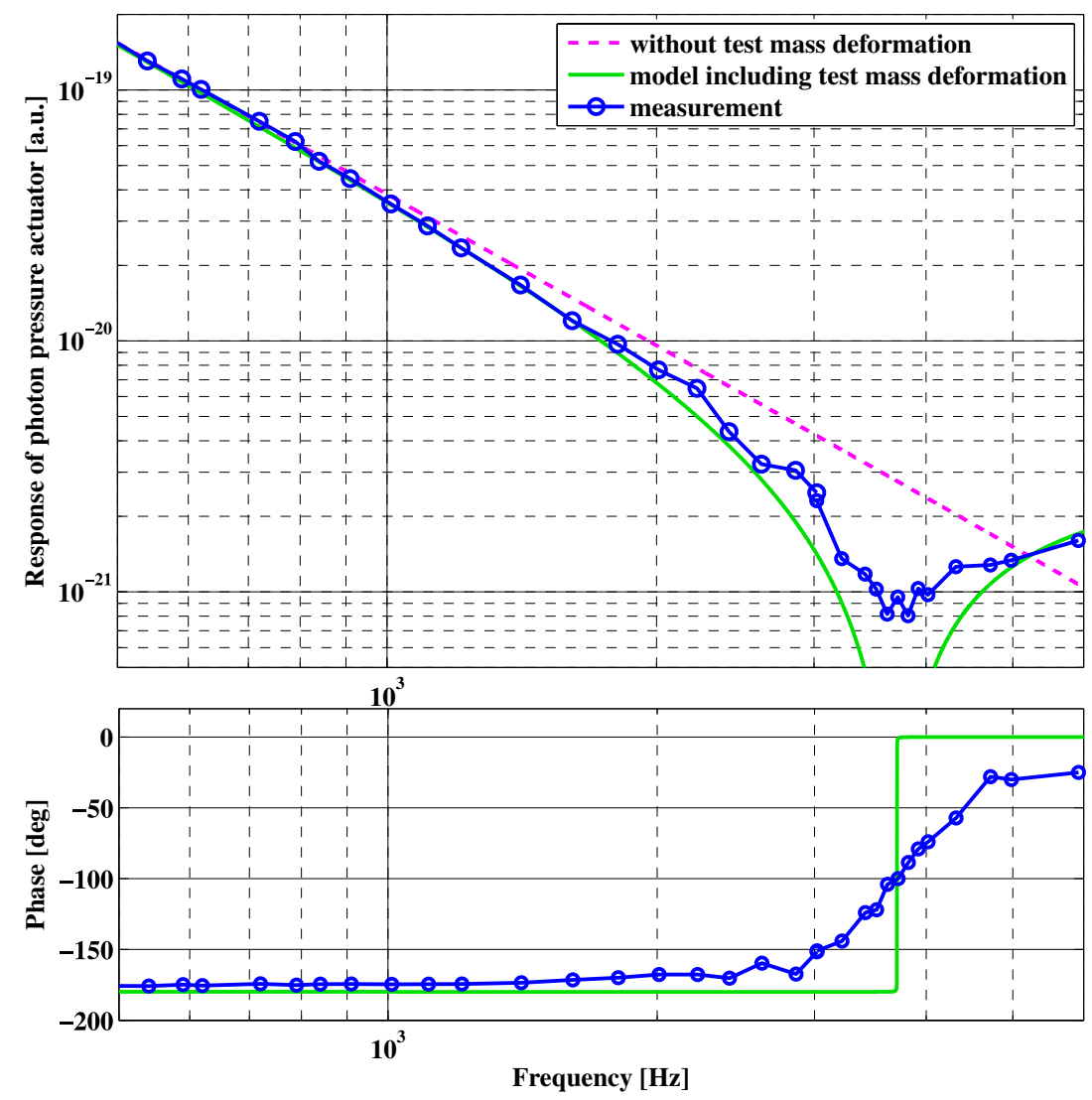

Figure 4. Result of the long duration photon pressure actuator injections over a wide frequency range. The pink dashed line represents the photon pressure actuator response without taking test mass deformation in account. The green solid line indicates the photon pressure actuator response taking the test mass deformation into account. The actual measurement of the response is displayed by the blue circles. The presence of the expected notch structure and by this the non-rigidity of the test masses in the detection band of the gravitational wave is clearly confirmed by the measurement.

notch structure. The measurements presented here include long duration measurements to achieve reasonable snr at high frequencies. Individual measurement points are made of single discrete Fourier transforms (DFT) containing up to $10 \mathrm{~h}$ of data. Such amounts of data are difficult to handle with standard computers. A heterodyne technique was employed to reduce the volume of data. The time series of the data containing the signal of interest, $E_{\mathrm{sig}} \cdot \sin \left(\omega_{\mathrm{sig}} t\right)$, is multiplied by a sine wave with a slightly lower frequency, $\omega_{\text {het }}$ :

$E_{\text {sig }} \cdot \sin \left(\omega_{\text {sig }} t\right) \cdot \sin \left(\omega_{\text {het }} t\right)=\frac{1}{2} E_{\text {sig }}\left[\cos \left(\omega_{\text {sig }}-\omega_{\text {het }}\right) t-\cos \left(\omega_{\text {sig }}+\omega_{\text {het }}\right) t\right]$.

The second term on the right-hand side of equation (5) still contains the signal, but shifted towards even higher frequencies. The signal component we are interested in is shifted to a very low frequency, $\left(\omega_{\text {sig }}-\omega_{\text {het }}\right)$, which for our investigations was chosen to be $9 \mathrm{~Hz}$. After heterodyning, the data stream is strongly low-pass filtered and down-sampled to give a data stream that can be handled by desktop computers. Figure 4 shows the result of these measurements. The pink dashed line represents the photon pressure actuator response without taking test mass deformation into account, while the green solid line indicates the 
photon pressure actuator response taking the test mass deformation into account. The actual measurement of the response is displayed by the blue circles.

The presence of the expected notch structure and by this the non-rigidity of the test masses in the detection band of the gravitational wave is clearly confirmed by the measurement. The magnitude of the response is about a factor of 3 below the pendulum response for frequencies between 3 and $4 \mathrm{kHz}$. At $5 \mathrm{kHz}$ the measurement matches the pendulum response and finally at $6 \mathrm{kHz}$ the measured response clearly exceeds the $1 / f^{2}$ behavior of the pendulum response. Around the notch frequency, the phase of the photon pressure actuator response also changes significantly from about $-165^{\circ}$ at $2.8 \mathrm{kHz}$ to about $-30^{\circ}$ at $4.8 \mathrm{kHz}$. A phase of nearly $0^{\circ}$ at high frequencies clearly indicates that the total response of the system is no longer dominated by the pendulum response.

It can be seen that the notch is smeared out in the experimental data. This originates from beam jitter of the main interferometer beam resulting in a varying overlap of the main interferometer beam and deformed test mass. Different overlaps correspond to different effective displacements

$$
D_{\text {total }}=r \cdot k_{I} \int_{0}^{0.09 \mathrm{~m}} \int_{0}^{360^{\circ}} \cdot I(r, \varphi) \cdot D(r, \varphi) \cdot \mathrm{d} r \cdot \mathrm{d} \varphi,
$$

which finally correspond to slightly shifted notch frequencies. Within the long measurement intervals of up to $10 \mathrm{~h}$ the measurement averages over a variety of notch frequencies resulting in a smeared out notch. A detailed description of this effect, including a quantitative analysis can be found in [7].

\section{Summary and conclusions}

We evaluated the deformation of a GEO 600 test mass caused by a narrow localized force, applied by a photon pressure actuator using finite element models as well as an analytical calculation. Based on these results, we developed a simple model showing that for frequencies above $1 \mathrm{kHz}$ the test mass deformation has a non-negligible effect on the response of the photon pressure actuator. Actual measurements confirmed the predictions of our model, in particular, around $3.8 \mathrm{kHz}$ the presence of a notch structure in the photon pressure actuator response is clearly observed in the measurements.

Test masses of the currently operating laser-interferometric gravitational-wave detectors cannot be considered to be rigid bodies for frequencies within the detection band. The effect of a test mass deformation needs to be taken into account when using the photon pressure actuation for high-precision calibration of any gravitational-wave detector. Furthermore, it should be mentioned that the actuation with widely used coil-magnet actuators can potentionally lead to test mass deformation effects as well.

\section{Acknowledgments}

The authors are grateful for the support from PPARC and the University of Glasgow in UK, and the BMBF and the state of Lower Saxony in Germany. LIGO was constructed by the California Institute of Technology and Massachusetts Institute of Technology with funding from the National Science Foundation and operates under agreement PHY-0107417. In addition, we would like to thank R Savage, P Kalmus, E Goetz, M Landry, B O'Reilly and the ILIAS collaboration for many fruitful discussions about the photon pressure calibrators. This paper has LIGO document number LIGO-P070074-00-Z. 


\section{References}

[1] Hild S et al 2006 The status of GEO 600 Class. Quantum Grav. 23 S643-51

[2] Acemese F et al 2004 Status of VIRGO Class. Quantum Grav. 21 S385-94

[3] Sigg D et al 2004 Commissioning of LIGO detectors Class. Quantum Grav. 21 S409-15

[4] Takahashi R (the TAMA Collaboration) 2004 Status of TAMA300 Class. Quantum Grav. 21 S403-8

[5] Clubley D A et al 2001 Calibration of the Glasgow $10 \mathrm{~m}$ prototype laser interferometric gravitational wave detector using photon pressure Phys. Lett. A 28385

[6] Mossavi K et al 2006 A photon pressure calibrator for the GEO600 gravitational wave detector Phys. Lett. A $3531-3$

[7] Hild S 2007 Beyond the first generation: extending the science range of the gravitational wave detector GEO 600 PhD Thesis University of Hannover

[8] www.ansys.com

[9] www.comsol.com

[10] Bondu F et al 1998 Thermal noise in mirrors of interferometric gravitational wave antennas Phys. Lett. A 246 227-36

[11] Liu Y T and Thorne K S 2000 Thermoelastic noise and homogeneous thermal noise in finite sized gravitationalwave test masses Phys. Rev. D 62122002

[12] Smith R et al 2004 Mechanical quality factor measurements of monolithically suspended fused silica test masses of the GEO600 gravitational-wave detector Class. Quantum Grav. 21 1091-8 